\title{
Laboratory astrophysics for the interpretation of stellar spectra
}

\author{
Ulrike Heiterㅁํ $†$ \\ Observational Astrophysics, Department of Physics and Astronomy, Uppsala University, \\ Box 516, 75120 Uppsala, Sweden
}

\begin{abstract}
High-resolution stellar spectra are important tools for studying the chemical evolution of the Milky Way Galaxy, tracing the origin of chemical elements, and characterizing planetary host stars. Large amounts of data have been accumulating, in particular in the optical and infrared wavelength regions. The observed spectral lines are interpreted using model spectra that are calculated based on transition data for numerous species, in particular neutral and singly ionized atoms. We rely heavily on the continuous activities of laboratory astrophysics groups that produce high-quality experimental and theoretical atomic data for the relevant transitions. We give examples for the precision with which the chemical composition of stars observed by different surveys can be determined, and discuss future needs from laboratory astrophysics.
\end{abstract}

Keywords. atomic data, stars: late-type, techniques: spectroscopic, surveys

\section{Introduction}

High-resolution stellar spectra play a crucial role for studying the chemical and dynamical structure and evolution of the Milky Way, deriving the origin of chemical elements, and determining the properties of planetary host stars. In recent years, large amounts of data have been accumulating from surveys and individual observing programs, in particular in the optical and infrared wavelength regions. The interpretation of the observed spectral lines using model spectra requires high-quality atomic transition data.

A number of ground-based observational surveys are currently obtaining and processing stellar spectroscopic data on an industrial scale, and several more are planned for the near future. The common goal of these surveys is to provide a homogeneous overview of the distributions of motions and chemical abundances in the Milky Way. The difference between surveys lies in the specific methods and/or objects studied in order to achieve this goal. We give three examples in chronological order, all of which are collecting spectra with a resolution of $\lambda / \Delta \lambda \gtrsim 20000$ for $10^{5}$ to $10^{6}$ stars.

The APOGEE survey (Majewski et al. 2017, USA), operating at infrared wavelengths (H-band), has been targeting 150000 stars with a focus on the dust-obscured parts of the Galaxy. The Gaia-ESO Public Spectroscopic Survey (Gilmore et al. 2012; Randich et al. 2013, ESO VLT)covers a considerable part of the optical spectral region and was designed to complement the ESA space mission Gaia by obtaining high-resolution spectra for up to 100000 faint stars. Also the GALAH survey (De Silva et al. 2015; Buder et al. 2018, Australia) is obtaining optical spectra (for up to $10^{6}$ stars), and has its focus on chemical tagging.

$\dagger$ and the Gaia-ESO line list group (Karin Lind, Maria Bergemann, Martin Asplund, Paul S. Barklem, Šarunas Mikolaitis, Thomas Masseron, Patrick de Laverny, Laura Magrini, Juliet C. Pickering et al.)

(C) The Author(s), 2020. Published by Cambridge University Press on behalf of International Astronomical Union. This is an Open Access article, distributed under the terms of the Creative Commons Attribution licence (http://creativecommons.org/licenses/by/4.0/), which permits unrestricted re-use, distribution, and 


\section{Line lists}

The atomic and molecular data needed for modelling stellar spectra by solving the equation of radiative transfer are usually compiled in a line list for each project. This is a list of (upper and lower) level energies, transition probabilities (often in the form of oscillator strengths or $g f$-values), and broadening parameters for each transition between two states (corresponding to a spectral line). Transition probabilities are taken either from measurements by laboratory astrophysics groups or from calculations by atomic physics groups. Line broadening is mainly caused by collisions with neutral or charged particles, for which very few experimental data have been obtained, and the majority of which are therefore calculations by atomic physics groups (Barklem 2016).

Data published by different laboratory astrophysics and atomic phsics groups may be extracted directly from the literature, or from databases specialising in different applications. Examples are the NIST Atomic Spectra Database (Kramida et al. 2018), the VALD database (Ryabchikova et al. 2015), and the STARK-B database (Sahal-Brëchot et al. 2017). The Virtual Atomic and Molecular Data Centre (Dubernet et al. 2016 and poster number 26 in this conference, VAMDC, http://www.vamdc.eu) is an electronic infrastructure providing access to $\sim 30$ databases simultaneously, both via a web interface (the VAMDC portal) and via various Virtual Observatory tools. When using data from databases care must be taken to include citations to the original literature in publications.

For the stellar spectroscopic surveys mentioned previously, the relevant species are mainly neutral and singly ionized atoms, as well as diatomic and triatomic molecules, as most targets for galactic and planetary studies are F-, G-, or K-type stars.

The line list used in the APOGEE survey (Shetrone et al. 2015) comprises $~ 130000$ lines for 36 atoms and 6 molecules in the wavelength range from 1500 to $1700 \mathrm{~nm}$, with the "best" atomic data from the literature, and astrophysical atomic data calibrated on the Sun and Arcturus for $\sim 20000$ lines. Within the Gaia-ESO consortium a large effort has been put into the construction of a line list which is being used as a source of common input data by the up to 14 research groups participating in the abundance analysis of the survey data. The Gaia-ESO line list has also constituted the starting point for the GALAH line list.

In brief, $\sim 1300$ transitions were preselected in the relevant wavelength ranges $(475 \mathrm{~nm}$ to $685 \mathrm{~nm}$ and $850 \mathrm{~nm}$ to $895 \mathrm{~nm}$ ), which were presumed to allow accurate determination of stellar parameters, and of abundances for many elements for FGK-type stars. A compilation of the best atomic data for these lines defined the standard line list, representing 35 elements (44 neutral and singly ionised species). A simple quality flag was assigned to each line, indicating whether the sources of $g f$-values were accurate recent laboratory measurements (published from the 1980s onwards), less accurate older experimental $g f$-values, or theoretical data. We emphasise that no astrophysical $g f$-values were included or derived.

The preselected lines were complemented with available data for all lines in the observed spectral range of the target stars, extracted from the VALD database in the case of atoms, and calculated and compiled by T. Masseron for 12 diatomic molecules. These data are needed to identify blends for the preselected lines, and as "background" for synthetic spectrum calculations. They were used to assign a second flag to each preselected line, according to blending properties.

As an example for an application of the Gaia-ESO line list, Fig. 1 shows observed (Hinkle et al. 2000) and calculated spectra for the giant star Arcturus in a small wavelength interval, convolved to the spectral resolution used in the Gaia-ESO survey. Most of the observed features are reproduced by the calculations, but deviations occur in several places. Calculated lines may be too weak or completely missing, or may be too strong 

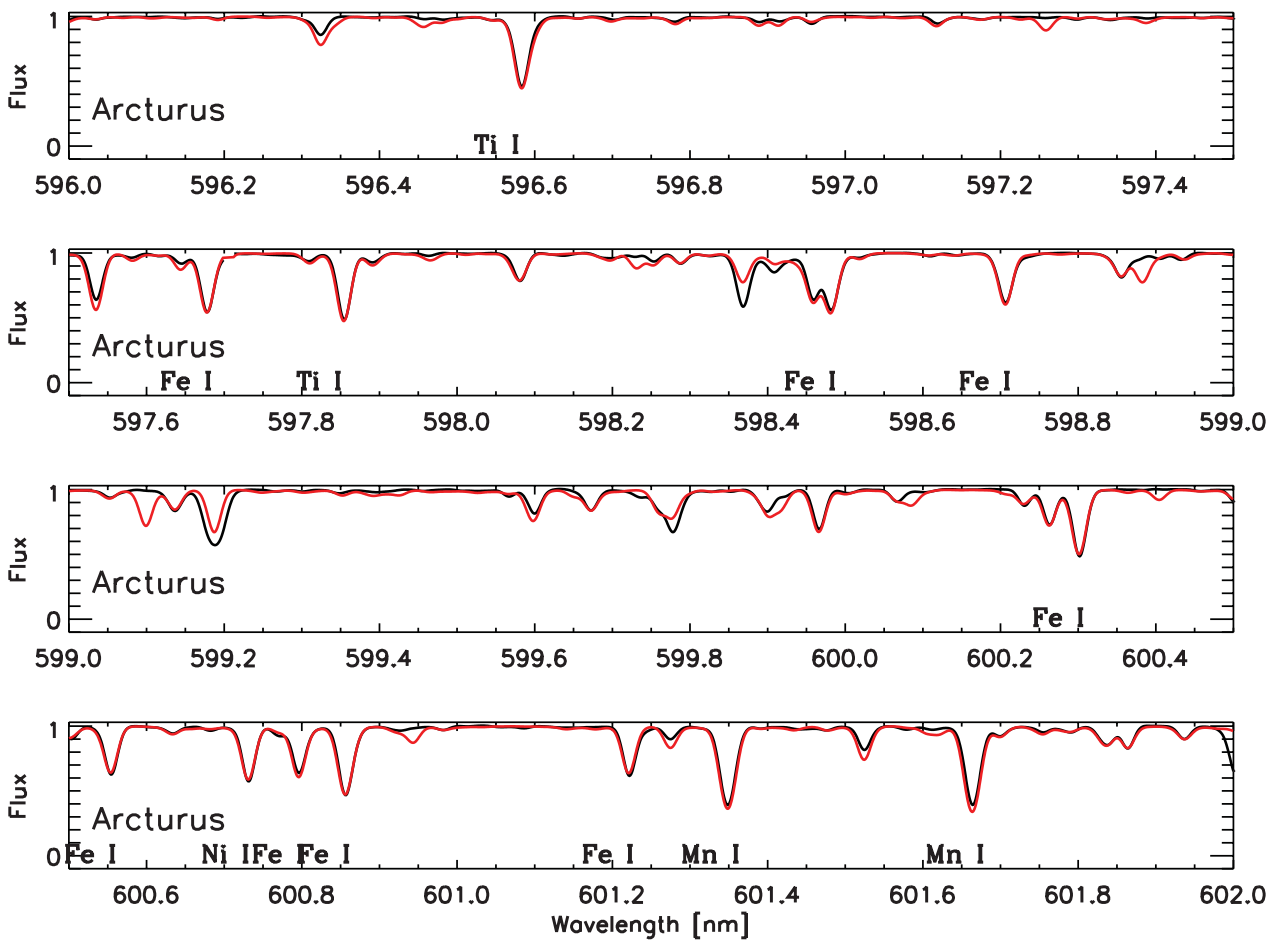

Figure 1. Observed (black) and calculated (red/gray) spectra for Arcturus for a small wavelength interval in the optical region. Some of the strongest lines from the Gaia-ESO line list are labelled by their species.

compared to the observed lines. This indicates deficiencies in the atomic data. An extensive description and discussion of the Gaia-ESO line list is to be found in Heiter et al., (in prep).

\section{Abundance precisions and data needs}

One way to evaluate the importance and quality of atomic data is to investigate the precisions of elemental abundances achieved by the different surveys. A caveat to this approach is however that the abundance precisions also depend on stellar parameters, analysis methods, and the definition of "precision". For example, in the APOGEE survey the star-to-star scatter within clusters is used, while the Gaia-ESO survey refers to the method-to-method dispersion or the line-to-line scatter.

Nevertheless, the general picture emerging is that the abundances obtained by the APOGEE survey (Holtzman et al. 2015; Mészáros et al. 2015; see also Holtzman et al. 2018; Jönsson et al. 2018) appear to achieve the highest precision (their uncertainties $<0.05$ dex) for $\alpha$-elements, $\mathrm{Fe}$, and $\mathrm{Ni}$, and the lowest precision for $\mathrm{V}$. Problems for $\mathrm{Al}$, $\mathrm{Ca}$, and $\mathrm{Ti}$ are encountered at low abundances.

Within the Gaia-ESO survey (Smiljanic et al. 2014; Mikolaitis et al. 2014; Lanzafame et al. 2015; Jofré et al. 2015) high precision abundances (uncertainties <0.15 dex) can be obtained for up to ten elements, including $\mathrm{Al}, \mathrm{Si}$, and $\mathrm{Ca}$, while the least reliable abundances are obtained for $\mathrm{Co}, \mathrm{Ni}, \mathrm{Zn}$, and $\mathrm{Y}$. There are also problems for $\mathrm{V}$ at low metallicities. 
The flags provided with the Gaia-ESO line list may be used to assess future data needs and to compile a wish list for new experimental $g f$-values in the optical wavelength region. Focussing on lines which are more or less unblended, high priority should be given to species which have uncertain transition probabilities for $>50 \%$ of these lines. This concerns $\sim 240$ Fe I lines, $\sim 50$ Ni I lines (whith high excitation energies), and some Fe II, NaI, Si I, and Ca II lines. However, there are a few species for which all of the few available "unblended" lines have uncertain $g f$-values, and these should be given even higher priority: $\mathrm{Al}$ I, SI, and CrII.

In summary, we emphasise the importance of accurate atomic data in the optical and IR for the interpretation of the data obtained by large-scale stellar spectroscopic surveys. Today, abundances with typical precisions of $\sim 0.1$ dex are being determined on an industrial scale for $\sim 10$ chemical elements. For recent progress in laboratory astrophysics the latest report by the IAU Working Group on High-Accuracy Stellar Spectroscopy (Barklem et al. 2018) may be consulted. The line-list work done within the Gaia-ESO survey provides a convenient starting point for the analysis of optical spectra of FGK stars. It also allowed us to identify several elements with urgent need for better atomic data.

This work of comprehensive data compiliation and quality assessment should be extended to the near-IR region in a similar way. Besides the APOGEE survey, highresolution spectra in the wavelength range from $\sim 700$ to $1400 \mathrm{~nm}$ are becoming ever more prominent sources of information about stars, for example in recent studies of the properties of M dwarfs (Lindgren \& Heiter 2017; Passegger et al. 2018).

\section{Acknowledgment}

U.H. acknowledges support from the Swedish National Space Agency (SNSA/ Rymdstyrelsen). Thanks go to RSC and STFC, and all sponsors of IAU S350.

\section{References}

Barklem, P. S. 2016, A\&\&A Rev., 24, 9

Barklem, P. S., Nahar, S., Pickering, J., Przybilla, N., \& Ryabchikova, T. 2018, Transactions of the IAU, Vol. XXXA, https://www.iau.org/static/science/scientific_bodies/working_ groups $/ 275 /$ wg-hass-triennial-report-2015-2018.pdf

Buder, S., Asplund, M., Duong, L., et al. 2018, MNRAS, 478, 4513

De Silva, G. M., Freeman, K. C., Bland-Hawthorn, J., et al. 2015, MNRAS, 449, 2604

Dubernet, M. L., Antony, B. K., Ba, Y. A., et al. 2016, J. Phys. B: At. Molec. Phys., 49, 074003

Gilmore, G., Randich, S., Asplund, M., et al. 2012, The Messenger, 147, 25

Hinkle, K., Wallace, L., Valenti, J., \& Harmer, D. 2000, Visible and Near Infrared Atlas of the Arcturus Spectrum 3727-9300 A (San Francisco: A SP) ISBN: 1-58381-037-4.

Holtzman, J. A., Hasselquist, S., Shetrone, M., et al. 2018, AJ, 156, 125

Holtzman, J. A., Shetrone, M., Johnson, J. A., et al. 2015, AJ, 150, 148

Jofré, P., Heiter, U., Soubiran, C., et al. 2015, A\&\&A, 582, A81

Jönsson, H., Allende Prieto, C., Holtzman, J. A., et al. 2018, AJ, 156, 126

Kramida, A., Yu. Ralchenko, Reader, J., \& and NIST ASD Team. 2018, NIST Atomic Spectra Database (ver. 5.6), [Online]. Available: https://physics.nist.gov/asd [Tue Oct 09 2018]. National Institute of Standards and Technology, Gaithersburg, MD. DOI:10.18434/T4W30F

Lanzafame, A. C., Frasca, A., Damiani, F., et al. 2015, A\& A, 576, A80

Lindgren, S. \& Heiter, U. 2017, A\& A, 604, A97

Majewski, S. R., Schiavon, R. P., Frinchaboy, P. M., et al. 2017, AJ, 154, 94

Mészáros, S., Martell, S. L., Shetrone, M., et al. 2015, AJ, 149, 153

Mikolaitis, S., Hill, V., Recio-Blanco, A., et al. 2014, A\& A, 572, A33

Passegger, V. M., Reiners, A., Jeffers, S. V., et al. 2018, A\&AA, 615, A6 
Randich, S., Gilmore, G., \& Gaia-ESO Consortium. 2013, The Messenger, 154, 47

Ryabchikova, T., Piskunov, N., Kurucz, R. L., et al. 2015, Phys. Scr, 90, 054005

Sahal-Bréchot, S., Dimitrijević, M. S., Moreau, N., \& Nessib, N. B. 2017, in American Institute of Physics Conference Series, Vol. 1811, Atomic Processes in Plasmas (APiP 2016), 030003

Shetrone, M., Bizyaev, D., Lawler, J. E., et al. 2015, ApJS, 221, 24

Smiljanic, R., Korn, A. J., Bergemann, M., et al. 2014, A\&\&A, 570, A122 
